\title{
Interferon-Induced Protein with Tetratricopeptide Repeats Family
}

National Cancer Institute

\section{Source}

National Cancer Institute. Interferon-Induced Protein with Tetratricopeptide Repeats

Family. NCI Thesaurus. Code C123809.

A family of proteins that are expressed during viral infection, pathogen recognition or during interferon (IFN) treatment. These proteins are localized in the cytoplasm and contain multiple tetratricopeptide repeats; they are involved in antiviral immune processes. 